\title{
A Review on High Burden of Malaria during Pregnancy: Need of Social Science Intervention
}

\author{
Bishwaranjan Purohit and Amarendra Mahapatra \\ Regional Medical Research Centre, Indian Council of Medical Research, \\ SE Rly Project Complex (PO), Bhubaneswar 751 023, Orissa, India \\ Telephone:0674-2303424, E-mail: purohitb@gmail.com
}

KEYWORDS Malaria. Pregnancy. Choloroquine. Preventive Intermittent Treatment

\begin{abstract}
Pregnant women and infants are more susceptible to malaria, and the malaria during pregnancy has deleterious effects on both the mother and the pregnancy outcome. In many malaria endemic countries, the preventive intermittent treatment (PIT) with sulphadoxine-pyrimithamine or chloroquine has been instituted. In India PIT with chloroquine has been instituted. Studies suggested that PIT prevent malaria during pregnancy and reduces the severe maternal anaemia, low birth weight babies and infant mortality substantially. Despite of availability of efficacious PIT with choloroquine, the treatment compliance rate is not satisfactory among several endemic communities including India. Hence, community's perceptions and utilization pattern of malaria chemoprophylaxis should be assessed properly and there is an urgent need of research towards the development of efficacious, cost effective and sustainable malaria control tools for protecting pregnant women. A strong social research component to study people's perceptions and knowledge towards understanding the lower utilization of PIT has to be incorporated in the PIT of the national programme. This research further helps the programme in developing 'culture-sensitive' community mobilization and communication strategies.
\end{abstract}

\section{INTRODUCTION}

Malaria is one of the major causes of morbidity and mortality in tropical regions like SouthAfrican and South-Asian countries. Because of the favourable and conducive environment, the parasite and the vector population sustain well in these regions. At present, more than 2000 million people are at the risk of the disease and 300-500 million people suffer from malaria each year. In India, about two million cases of malaria were reported annually. Although the population of Orissa state represents only 4 percent of the total population of India, it contributes 22 percent of all malaria cases, 43 percent of all cases of Plasmodium falciparum malaria and 50 percent of all malaria attributable deaths reported in the country (Sharma et al. 1996)

After launching of the National Malaria Eradication Programme (NMEP) in 1958, a considerable reduction in the incidence of malaria was observed in the state as in other parts of the country. But after 1965, a resurgence of malaria

Address for correspondence: Bishwaranjan Purohit, Regional Medical Research Centre, Indian Council of Medical Research, SE Rly Project Complex (PO)

Bhubaneswar 751 023, Orissa, India

Telephone: 0674-2303424

E-mail:purohitb@gmail.com occurred in different parts of the country and Orissa was no exception. Malaria control in Orissa was logistically difficult to operate mainly due to three-fourths of the state being mountainous and forested, relatively inaccessible with poor communication and inadequate health care facilities. The climate of Orissa is very much favourable for mosquitoes to grow. The average number of rainy days is $60-90$ days per year. The mean annual rainfall is $1482 \mathrm{~mm}$ but the maximum is during JuneSeptember. The mean maximum temperature in the state is $32.8^{\circ} \mathrm{C}$, which rises up to $45^{\circ} \mathrm{C}$ in AprilMay; and the mean minimum temperature is $22.8^{\circ} \mathrm{C}$, which falls to $11^{\circ} \mathrm{C}$ in December; and the mean range of relative humidity is $61.5-85.7 \%$ in the morning and $52.8-80.4 \%$ in the evening. The optimum temperature of $20-30^{\circ} \mathrm{C}$ and relative humidity of $60 \%$ are favourable and for the development of the malaria parasite in the mosquito for mosquitoes to live their normal life span (Ranjit 2006).

\section{PREGNANCY AND MALARIA}

Each year, approximately 50 million women living in malaria-endemic countries throughout the world become pregnant. An estimated 10,000 of these women and 200,000 of their infants die as a result of malaria during pregnancy, and severe 
malarial anaemia contributes to more than half of these deaths (WHO 2007a). Pregnant women and newborn children are the worst sufferer of malaria as these two groups are highly susceptible to malaria infection (Singh et al. 2005). For instances, malaria during pregnancy in sub-Saharan Africa was estimated to account for: 400,000 cases of severe anaemia in pregnant women, $\sim 35 \%$ of preventable low birth weight, $\sim 5 \%$ of infant mortality and 75,000-200,000 infant deaths annually (CDC 2004). Malaria kills 1.5 to 2.7 million persons every year and adversely affects another 3000,000 to 5000,000, mostly in Africa (WHO 2000, 2003a). Pregnant women are vulnerable to malaria as pregnancy reduces a woman's immunity to malaria, making her more susceptible to infection and increasing risk of severe anaemia and death. For the foetus, maternal malaria increases the risk of spontaneous abortion, stillbirth, premature delivery and low birth weight (WHO 2007b). Again pregnant women are especially prone to severe attacks of malaria, which may cause abortion, premature labour and still birth (Clark 1915; Blacklock and Gordon 1925). Up to 200,000 newborn deaths years are thought to be due to malaria in pregnancy. Malaria during pregnancy causes as many as 10,000 maternal deaths each year, 8-14 percent of all low birth weight babies, and 3-8 percent of all infant deaths in certain parts of Africa (WHO 2007b). In India very few studies were carried out, so far in the field of malaria during pregnancy, and the epidemiological data on pregnancy related malaria not available from India. Hence, this study attempts to highlight the problems of pregnancy related malaria and warrants to develop feasible strategies to deal with the problems.

In addition, malaria has also more pregnancy related problems like foetal wastage, prematurity, dysmaturity, stillbirth and congenital malaria, jaundice, malnutrition, etc. among children. Again, women with semi-immunity to malaria who live in regions where the disease is endemic are at increased risk for more frequent and severe episode of malaria during pregnancy (Ramharter et al. 2005). It's also proved that the prevalence of infection in the forest areas of India was highest in the young children (aged 1-5 years) and gradually declined with increasing age (Sharma et al. 2004). Thus malaria is widely recognized as an infection, which can seriously jeopardize the outcome of pregnancy. Where the disease is epidemic or have low endemicity, women of all parities seem equally affected (McGregor 1984) and symptomatic malaria is seen at all ages (Nosten et al., 1991). Again placental malaria is closely linked with anaemia, low birth weight (LBW) and decreasing venous blood in hypo endemic malarial area. Malaria is one of the most important causes of LBW in first pregnancies in Africa (Sarr et al. 2006). Mean haemoglobin concentrations were significantly lower in the infants who had been LBW babies than in the others, and significantly associated with parities, peripheral parasitaemia at delivery and placental malaria (Verhoeff et al. 1999).

Thus malaria infection during pregnancy is a major public health problem in tropical and Subtropical regions throughout the world. Pregnant women are the main adult risk group for malaria. In areas of epidemic or low (unstable) malaria transmission, adult women have not acquired any significant level of immunity and usually become ill when infected with malaria. For pregnant women in these areas the risk of developing severe malaria is 2-3 times higher than that for non-pregnant women living in the same area. Maternal death may result either directly from severe malaria or indirectly from malariarelated severe anaemia. In addition, malaria may result a range of adverse pregnancy outcomes, including low birth weight, spontaneous abortion, and neonatal death (WHO 2003b). Most studies come from sub-Saharan Africa, where approximately 25 million pregnant women are at risk of malaria infection every year, and one in four women have evidence of placental infection at the time of delivery.

From India, very little information is available on the relationship between malaria infection and pregnancy with respect to birth outcome in Indian subcontinent, except one hospital based investigation on 365 pregnant women in central India (Singh et al. 1999). This study concluded that mean parasite densities were significantly higher in pregnant women compared with nonpregnant women for both Plasmodium falciparum and Plasmodium vivax infection. Again, pregnant women with falciparum or vivax malaria were significantly more anaemic than noninfected pregnant women or infected nonpregnant women. With regard to the LBW, $89 \%$ of babies weighted $<2.5 \mathrm{~kg}$ in the infected group compared with $38 \%$ in the control group. This study also showed high malaria prevalence among pregnant women, which was statistically 
significant compared with the situation among non-pregnant women. Cerebral malaria was a common complication of severe Plasmodium falciparum infection, with a high mortality during pregnancy (Singh et al. 1999).

Thus malaria in pregnancy is a major concern of the public health problem. Controlling this problem needs better control over the disease and reforms in health care systems. The increased rate of maternal mortality rate (MMR) and infant mortality rate (IMR) are the outcomes of poor health care of mother (pregnant mother) and infants respectively. And poor socio-economic condition is too related to malaria burden and vice-versa (Sinton 1935)

\section{PREVENTIVE INTERMITTENT TREATMENT (PIT) AND MALARIA CHEMPROPHY LAXIS COMPLIANCE}

In many endemic countries including India, the preventive intermittent treatment (PIT) has been instituted by the government health systems. Chloroquine or sulphadoxine-pyrimithamine (SP) is being distributed to the pregnant women intermittently during their pregnancy to safeguard the mother and child. PIT of malaria reduces the bad health consequences due to malaria of mother and child. In Malawi, where the therapy has been part of national health policy since 1993, women receiving PIT with SP during their pregnancy had significantly lower rates of placental infection, reduced from $32 \%$ to $23 \%$, and a lower rate of low birth weight babies, a reduction from $23 \%$ to $10 \%$ (WHO, 2007b). PIT with SP tried in Gabon, there was a marked benefit on the prevalence of low birth weight and premature birth for women adhering to national recommendations i.e. PIT (Ramharter 2007). In another study in Nigeria, PIT with SP has prevented the maternal and placental malaria in parturient mothers (Falade et al., 2007). Still this programme needs further strengthening at grass root programme and policy level, keeping in view the knowledge, perceptions and health seeking behaviour of the community. The new approaches increased access to and compliance with PIT (Mbonye et al. 2007).

As per the safety of chemoprophylaxis intake is concerned, it is concluded from a study that pregnancy exposed to quinine or choloroquine and carried to term did not have increased rates of congenital abnormality, stillbirth or LBW. These results suggest that therapeutic doses of quinine and choloroquine are safe to use even during the first trimester of pregnancy (McGready et al. 2002).

So, above studies shows the malaria burden over pregnant women and infants, and also the severity of pregnancy related outcomes contributed by malaria. Still the malaria drug compliance rate is not appreciable. The nonacceptance rate to the drug is significantly diminishing among them, more surprisingly among the pregnant women. This is due to several factors, like efficacy of the intervention, provider acceptability, cost effectiveness, safety, lack of proper co-ordination between the local health care system and the community, etc. Though steps are being taken by the policy makers and government to control malaria by distributing choloroquine tablets during pregnancy, no success is witnessed. Similarly in a study, failure of a choloroquine chemoprophylaxis programme to adequately prevent malaria during pregnancy is concluded in Burkina Faso (Sirima et al. 2003).

For a successful PIT programme, compliance pattern is very much important. Before undertaking a programme, proper sensitization of the community is necessary so as to make the programme successful. Similarly, vulnerability of pregnant women towards malaria and its bad delivery outcome has to be understood by the community. Community participation in this regard has to be promoted. For example compliance to malaria chemoprophylaxis among pregnant women in Malawi has historically been low. Three separate interventions, based upon an ethno-graphic study of malaria beliefs among pregnant women in Malawi, were introduced to increase compliance to the malaria chemoprophylaxis program provided by the Ministry of Health. Each intervention consisted of a health education messages (HelitzerAllen et al. 1993). In the study, a community-based formative research to learn about the local concept of malaria and issues surrounding malaria prevention and treatment during pregnancy has been undertaken. The resulting interventions were tested in four clinics, and included a change in the health education message given during antenatal sessions, distribution of a sugar-coated choloroquine tablet, and an intervention combining the two strategies. The results showed a $45 \%$ increase in choloroquine use when the health education message was changed, and a $64 \%$ increase when the product was changed (Helitzer-Allen et al. 1994).

Similar attempt to study the reasons of non- 
compliance is a necessary to support the on going programme. Ethnographic study in view of community perception, utilization, knowledge, attitude, and practices to make conclusive feed back for better intervention is necessary to make the strategy successful at programme level. Currently in Africa, one of the main strategies to reduce malaria infection during pregnancy is the promotion of PIT. To date only a few studies have investigated the factors affecting compliance to PIT. A medical anthropological study described these factors from the perspective of pregnant women in rural Malawi. It concluded that understanding of the multiple contexts affecting malaria prevention is important, and that ethnographic research is useful for discovering and solving problems beyond the scope of many other research approaches (Launiala and Honkasalo 2007). But very few studies have been made (only in African countries) in view of PIT, compliance pattern, community behaviour, utilization, etc, and such studies are essentials to make future strategies by the planners and policy makers over from India. Apart from that, community based suggestions for betterment of the programme is beneficial for the change in strategies. This in turns come up with new interventions acceptable to the community. One such study on efficacy of choloroquine chemoprophylaxis is being carried out in Orissa, India (Mahapatra et. al. 2006). This study reports that $600 \mathrm{mg}$ of weekly choloroquine is given as PIT in Orissa, and a compliance rate of $36 \%$ among a cohort of pregnant women. The successful implementation of the PIT during pregnancy strategy depends on the proper planning of, and support to, the training of health staff and sustained sensitization of pregnant women at health facility and community levels about the benefits of PIT during pregnancy (Mubyazi et al. 2005).

\section{ROLE OF SOCIAL SCIENCE}

Thus, these problems have raised concerns among the researchers to find out solutions, which need in-depth research in this field to put forward options for the policy makers. This would provide improved strategies facilitating more access for the community. More access means to find out new dimensions through more compliance and acceptability of the drug. For which over all change is needed in indicators like, sensitization and awareness among the community, health education, community behaviour, and health care approach to the pregnant women, health care system with respect to community, proper co-ordination between drug provider and the community, finally community acceptance to the drug and health care approach in its sociocultural context. A socio-cultural understanding of the implementation context is prerequisite for planning meaningful programme for the pregnant women (Launiala and Kulmala 2006). The successful implementation of the PIT during pregnancy depends on the proper planning of, and support to, the training of health staff and sustained sensitization of pregnant women at health facility and community levels about the benefits of PIT for the women and their unborn babies (Mubyazi et al. 2005).

The priority areas for current research area on malaria during pregnancy are to search for efficacious, cost effective and sustainable malaria control tools (Menendez 1999). In depth ethnographic research at community level is essential to find out scope, gaps, and lacunas of above factors, which in turn would help the policy makers to put a new approach with improved and reformed strategies. Another major problem of programme managers and implementers is that how to translate the information produced by scientific research into feasible and sustainable programme mode. The total of our current knowledge needs to be assembled and regularly reviewed, as a basis for promoting the best strategies available and for identifying important gaps in our knowledge. The choice of best strategies will only be rational if the scientists collaborate more closely with the policy-makers and health managers. This collaboration involves the evaluation of current practices as well as the promotion of new ones (D'Alessandro 1999).

As malaria is one of the major public health problems of India, there is an urgent need to study or assess people's perception and utilization pattern of malaria chemoprophylaxis programme of the National Vector Born Disease Control Programmer's PIT with choloroquine among the pregnant women. Research on and implementation of malaria control intervention for pregnant women have predominantly ignored community responses or, when considered, they have centered on single, isolated factors usually with the aim of designing "culturally-sensitive" information, education, and communication 
messages. Reality, however, shows that the implementation of "simple" tools, like PIT to pregnant women, is not so simple because community reactions are not taken into account. With this article, it is expected to create awareness among researchers of such complex interactions and of the need of involving social sciences even for apparently "straight forward" interventions. Studying these interactions may help to improve the delivery of adequate interventions and thus contribute to attaining the target of reaching maximum number of pregnant women to protect them against malaria infection and its consequences. These models have to be taken as a first contribution to cover social science aspects. Such models will help to inspire future works in the malaria social science research, particularly in the under-researched field of malaria and pregnancy.

\section{REFERENCES}

Blacklock DB, Gordon RM 1925. Malaria infection as it occurs in late pregnancy, its relationship to labour and early infancy. Ann Trop Med Parasitol, 19: 327365 .

CDC 2004. Malaria. Atlanta, USA: Center for Disease Control and Prevention.

Clark HC 1915. The diagnostic value of the placental blood film in aestivoautumn malaria. J Exp Med, 22: 427-445.

D’Alessandro U 1999. A rational of approach to malaria control in pregnancy in Sub-Saharan Africa; the need for a link between scientific research and pub-health. Intervention. Ann Trop Med Parasitol, 93, Suppl 1: $575-577$

Falade CO, Yusuf BO, Fadero FF, Mokuolu OA, Hamer $\mathrm{DH}$, et al. 2007. To evaluate the effectiveness of IPT-SP in the prevention of maternal and placental malaria in parturient mothers in Ibadan, Nigeria. Malaria J, 6: 88

Helitzer-Allen DL, Macheso A, Wirima J, Kendall C 1994. Testing strategies to increase use of choloroquine chemoprophylaxis during pregnancy in Malawi. Acta Trop, 58(3-4): 255-266.

Helitzer-Allen DL, McFarland DA, Wirima JJ, Macheso AP 1993. Malaria chemoprophylaxis compliance in pregnant women: a cost-effectiveness analysis of alternative interventions. Soc Sci Med, 36(4): 403407.

Launiala A, Honkasalo ML 2007. Ethnographic study of factors influencing compliance to intermittent preventive treatment of malaria during pregnancy among Yao women in rural Malawi. Trans $R$ Soc Trop Med Hyg, 101(10): 77-94.

Launiala A, Kulmala T 2006. The importance of understanding the local context: Women's perceptions and knowledge concerning malaria in pregnancy in rural Malawi. Acta Trop, 98: 111-117.

Mahapatra A, Purohit B, Hansdah DP 2006. Annual
Report-2006, Bhubaneswar: Regional Medical Research Center.

Mbonye AK, Magnussen P, Bygbjerg IB 2007. Intermittent preventive treatment of malaria in pregnancy: the effect of new delivery approaches on access and compliance rates in Uganda. Trop Med Int Health, 12 (4): 519-531.

McGready R, Thwai KL, Cho T, Samuel LS, White NJ, et al. 2002. The effects of quinine and choloroquine for anti malarial treatment in the first trimester of pregnancy. Trans R Soc Trop Med Hyg, 96 (2): 180184.

McGregor IA 1984. Epidemiology, malaria and pregnancy. Am J Trop Med Hyg, 33 (4): 517-525.

Menendez C 1999. Priority areas for current research on malaria during pregnancy. Ann Trop Med Parasitol, 93 Suppl 1: S71-S74.

Mubyazi GM, Bloch P, Kamugisha ML, Kitua AY, Ijumba J 2005. Intermittent preventive treatment of malaria during pregnancy: a qualitative study of knowledge, attitudes and practices of district health managers, antenatal care staff and pregnant women in Korogwe District, North-Eastern Tanzania. Malaria J, 4:31.

Nosten F, ter Kuile F, Maelankirri L, Decludt B, White NJ 1991. Malaria during pregnancy in an area of unstable endemicity. Trans $R$ Soc Trop Med Hyg, 85(4): 424-429.

Ramharter M, Grobusch MP, Kiessling G, Adegnika AA, Möller U, et al. 2005. Clinical and parasitological characteristics of puerperal malaria in a study from Lambarene, Gabon. J Infect Dis, 191: 1005-1009.

Ramharter M, Katharina S, Marielle KB, Ayola AA, Kristen S, et al. 2007. Malaria in Pregnancy Before and After the Implementation of a National IPTp Program in Gabon. Am J Trop Med Hyg, 77(3): 418-422.

Ranjit M R 2006. The epidemiology of malaria in Orissa. ICMR Bull, 36(6-7): 1-10.

Sarr D, Marrama L, Gaye A, Dangou JM, Niang M, et al. 2006. High prevalence of placental malaria and low birth weight in sahelian periurban area. Am J Trop Med Hyg, 75: 171-177.

Sharma R S, Sharma GK, Dhillon GPS 1996. Epidemiology and Control of Malaria in India. Delhi: National Malaria Eradication Programme.

Sharma SK, Tyagi PK, Padhan K, Adak T, Subbarao SK 2004. Malarial morbidity in tribal communities living in the forest and plain ecotypes of Orissa, India. Ann Trop Med Parasitol, 98(5): 459-468.

Singh N, Saxena A, Awadhia SB, Shrivastava R, Singh MP 2005. Evaluation of a rapid diagnostic test for assessing the burden of malaria at delivery in India. Am J Trop Med Hyg, 73(5): 855-858.

Singh N, Shukla MM, Sharma VP 1999. Epidemiology of Malaria in pregnancy in central India. Bull World Health Organ, 77 (7):567-572.

Sinton JA 1935. What malaria costs India, nationally, socially and economically? Records of the Malaria Survey of India, 5: 233-264.

Sirima SB, Sawadogo R, Moran AC, Konate A, Diarra A, et al. 2003. Failure of a choloroquine chemoprophylaxis programme to adequately prevent malaria during pregnancy in Koupela District, Burkina Faso. Clin Infect Dis, 36(11): 1374-1382.

Verhoeff FH, Brabin BJ, Chimsuku L, Kazembe P, Broadhead RL 1999. Malaria in pregnancy and its consequences for the infant in rural Malawi. Ann 
Trop Med Parasitol, 93 Suppl 1: S25-S33.

WHO 2000. World Health Organization Expert

Committee on Malaria: Twentieth Report.

Geneva:World Health Organization.

WHO,2003a. World Health Organization and United

Nations Children's Fund. Africa malaria report,

Retrieved Jan 11, 2007 from <http://www.rbm.

who.int/amd2003/amr2003/amr_toc.htm>

WHO 2003b. Africa Malaria Report-Malaria during

pregnancy, World Health Organization, Geneva,
Retrieved Feb 15, 2007 from <http://www.rbm.who. int/amd2003/amr2003/ch4.htm>

WHO 2007a. Global Malaria Programme-Pregnant Women and Infants. World Health Organisation, Geneva, Retrieved Feb 18,2007 from <http://www. who.int/malaria/pregnantwomenandinfants.html> WHO 2007b. Roll Back Malaria-Malaria in pregnancy. World Health Organization, Geneva, Retrieved Feb 26, 2007 from <http://rbm.who.int/cgi-bin/rbm/ rbmportal/custom/rbm/home.do> 